\title{
THE DAMAGE OF THE OPTIC NERVE AS THE OUTCOME OF UNINTENTIONAL POISONING BY CLOSANTEL
}

\author{
Ioyleva Elena Eduardovna ${ }^{1}$, Kurakina Evgenia Sergeevna ${ }^{1}$, Saif Mutlaq Ali Saif ${ }^{1}$, Gavrilova Natalia Alexandrovna ${ }^{1}$ \\ Correspondence: kolkas2008@mail.ru \\ ${ }^{1}$ S.N. Fedorov NMRC, MNTK, Eye Microsurgery, Moscow, Russia
}

\section{Article History: \\ Received: September 19, 2019 \\ Accepted: December, 12020 \\ Published: January 1, 2021}

\section{Cite this as:}

Ioyleva EE, Kurakina ES, Saif

SMA, Gavrilova NA. The damage

of the optic nerve as the outcome

of unintentional poisoning by

closantel. Malang Neurology

Journal; 2021.7:70-73

http://dx.doi.org/10.21776/ub.mnj

.2021 .007 .01 .14

\section{ABSTRACT}

Background: In recent decades, the problem of toxic damage of the visual system due to poisoning by various medicines has become particularly relevant. The clinical case of a bilateral acute optic neuropathy of toxic genesis in a patient by the unintentional application of closantel used in veterinary practice is presented in the report. Closantel is a structural derivative of the salicylanilides, which is used exclusively in veterinary medicine for the prevention and treatment of large and small-scale cattle. Neurological and ophthalmic symptoms are often associated with the intoxication of closantel.

Objective: To describe the toxic damage of the optic nerve due to unintentional poisoning by closantel.

Methods: A patient aged 55 years is examined. A standard survey of ophthalmology was carried out. Photoregistration of the ocular fundus was used by a funduscamera Visucam 500 (Carl Zeiss Meditec AG, Germany), from special methods of research - the spectral optical coherence tomography (OCT) with a study of the macular area of the retina and the disc of the optic nerve on the device Zeiss Cirrus 5000 (Carl Zeiss Meditec, USA) and the optical coherence tomography with the function of angiography (OCT-A) of the macular area of the retina using RTVue XR Avanti (Optovue, USA)

Results: The patient complained of a severe, yet painless, reduction in vision of both eyes after an accidental intramuscular injection of the medicine called "Closantrem" in dose $4.0 \mathrm{ml}$. The visual acuity OU - an accounting of fingers on the face at a distance of $10 \mathrm{~cm}$. By ophthalmoscopy OU: the disc of the optic nerve is discolourated, its boundaries are indistinct, the arteries and the veins are narrowed, in the macular area of the retina without a visible physical pathology. The perimetry is in the correct projection of the light. A reduction in the thickness of the external and internal layers of the retina was observed as a result of the OCT of the macular zone OU. According to OCT of the disc of the optic nerve: the thickness of the layer of nerve fibres is in the upper and lower nasal segments. $\mathrm{OS}$ - the prominence of the disc of the optic nerve in vitreous. The fascinated changes of the cerebral cortex have not been found during the MRT. The diagnosis was made OU: Acute optic neuropathy of toxic genesis. A course of integrated conservative treatment has been introduced: the antiinflammatory, metabolic, nootropic, vitamin therapy, magnetotherapy, electrostimulation and electrophoresis.

Conclusion: Potentially, any medicines are toxic, which may cause a number of side effects from the visual and central nervous system. The toxic damage of the ganglion cells of the retina was initially occurring due to unintentional application of closantel, resulting in a reduction of the central visual acuity, followed by the development of the optic nerve atrophy.

Keywords: Closantel, visual system, toxic optic neuropathy, optic nerve atrophy, optical coherence tomography.

\section{Introduction}

In recent decades, the problem of acute and chronic poisonings has become particularly relevant as a result of the accumulation of a huge amount of various chemicals in the natural world, about 6 million names. ${ }^{11}$

Among the accidents in which emergency medical care is required, there are now quite frequent poisonings of medicines taken in a dose above the treatment. They are different from allergic reactions, side effects, individual intolerance, which are usually dependent on the body's characteristics and may occur even after the treatment of a therapeutic dose of the medicine.
Halogenated salicylanilides are a large group of compounds developed mainly for their antiparasitic activity by animals. Closantel is a structural derivative of the salicylanilides, which is used exclusively in veterinary medicine for the prevention and treatment of large and small-scale cattle. The method of action of the preparation is to change the processes of phosphorylation and transport of electrons, resulting in the disruption of the exchange processes and the loss of the parasite. ${ }^{4}$

In Russian Federation, a number of preparations have been registered as «Closantrem», «Santel», «Santomectin», «Rolenol», «Closantex» and «Closalben». Closantrem is a moderately hazardous substance to the extent of exposure to the organism. It is well tolerated by animals in 
recommended doses, and does not have a local irritating, embryotoxic, teratogenic, mutagenic and sensitizing effect.

Neurological and ophthalmic symptoms are often associated with the intoxication of closantel. The central nervous system, the retina and the optic nerve are usually affected. $^{11}$ It has been found that this preparation has neurotoxicity, which is capable of causing spongiosis, the loss of visual neurons, as well as neurons of the central nervous system and the spine. ${ }^{10}$

At present, only 4 cases are known for the toxicity of closantel in humans.4,6 The outbreak of toxicity of this medicine was first described in Lithuania in the 1990s.4 Two sporadic cases were also registered in Morocco.,

The unintentional application of the closantel was the cause of toxicity, which led to blindness in these reports. Partial restoration of vision with plasma exchange was reported in humans who swallowed a closantel for fear of helminthiasis. ${ }^{6}$

Objective. To describe the toxic damage of the optic nerve due to unintentional poisoning by closantel.

\section{Methods}

The A patient aged 55 years is examined. A standard survey of ophthalmology was carried out (vizometry, perimetry, pneumotonometry, biomicroscopy, ophthalmoscopy, electrophysiology survey). Photoregistration of the ocular fundus was used by a funduscamera Visucam 500 (Carl Zeiss Meditec AG, Germany), from special methods of research - the spectral optical coherence tomography (OCT) with a study of the macular area of the retina and the disc of the optic nerve on the device Zeiss Cirrus 5000 (Carl Zeiss Meditec, USA) and the optical coherence tomography with the function of angiography (OCT-A) of the macular area of the retina using RTVue XR Avanti (Optovue, USA).

Scanning of the particular area was carried out by protocol (Macular Cube 512x128), scanning of the area of the disc of the optic nerve - according to the protocol, the study of the parameters of the head of the optic nerve (Optic Disc Cube 200*200).

This study followed the tenets of the Declaration of Helsinki and all privacy requirements were met.

\section{Results}

The patient complained of a severe, yet painless, reduction in vision of both eyes after an accidental intramuscular injection of the medicine called "Closantrem" in dose 4.0 $\mathrm{ml}$. The man sought for the medical care with these complaints, the signs of intoxication (the headaches, the vomiting, the diarrhea) and the neurological disorders (the mild muscle weakness, the dyslexia) to the district hospital at the place or residence.

It was excluded the poisoning of the botulinum toxin by examination. A standard survey of ophthalmology was performed (the visual acuity OU - 0,005 (not corrected), the intraocular pressure OD $10 \mathrm{~mm} \mathrm{Hg}$, OS $6 \mathrm{~mm} \mathrm{Hg}$, the movement of the eyes in full). By biomicroscopy: both eyes are calm, the conjunctivae are pale pink, the corneas are transparent, the front chambers of the eyes are of medium depth, the lenses are transparent. By ophthalmoscopy OU: the disc of the optic nerve is monotonous, its boundaries are indistinct,

the size of the receptacles has been modified (the arteries are thread-shaped, the veins are narrowed (1:2)), in the macular area of the retina is the flattened reflex. The perimetry is in the correct projection of the light. A reduction in the thickness of the external and internal layers of the retina and the atrophy of a complex of the ganglions cells of retina were observed as a result of the OCT of the macular zone OU. According to OCT of the disc of the optic nerve: the prominence of the disc of the optic nerve in vitreous. The diagnosis was made OU: Acute toxic retrobulbar neuritis.

The conservative treatment has been introduced: a course of the anti-inflammatory, the detoxification, the angioprotective and the neuroprotective therapy. When the patient is discharged, the visual acuity OU - 0,01 (not corrected).

In a month no positive dynamics was observed after the treatment, the patient was referred to S.N. Fedorov NMRC «MNTK «Eye Microsurgery»». A standard survey of ophthalmology was performed (the visual acuity OU - an accounting of fingers on the face at a distance of $10 \mathrm{~cm}$, the intraocular pressure OD $10 \mathrm{~mm} \mathrm{Hg}$, OS $9 \mathrm{~mm} \mathrm{Hg}$, the movement of the eyes in full). By biomicroscopy: both eyes are calm, the conjunctivae are pale pink, the corneas are transparent, the front chambers of the eyes are of medium depth, the lenses are transparent. By ophthalmoscopy OU: the disc of the optic nerve is discolourated, its boundaries are indistinct, the arteries and the veins are narrowed, in the macular area of the retina without a visible physical pathology. The perimetry is in the correct projection of the light.

From special methods of research were carried out the spectral optical coherence tomography with a study of the macular area of the retina and the disc of the optic nerve on the device Zeiss Cirrus 5000 (Carl Zeiss Meditec, USA) (Figure 1, 2).
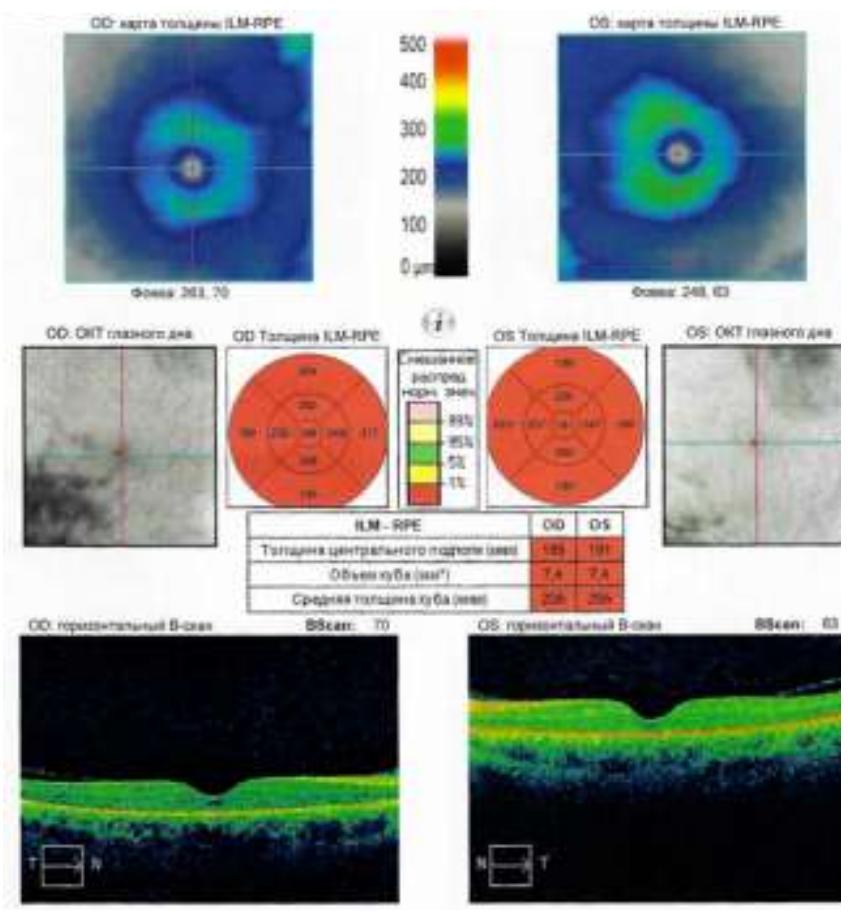

Figure 1. OCT of the macular zone OU: a reduction in the thickness of the external and internal layers of the retina. 


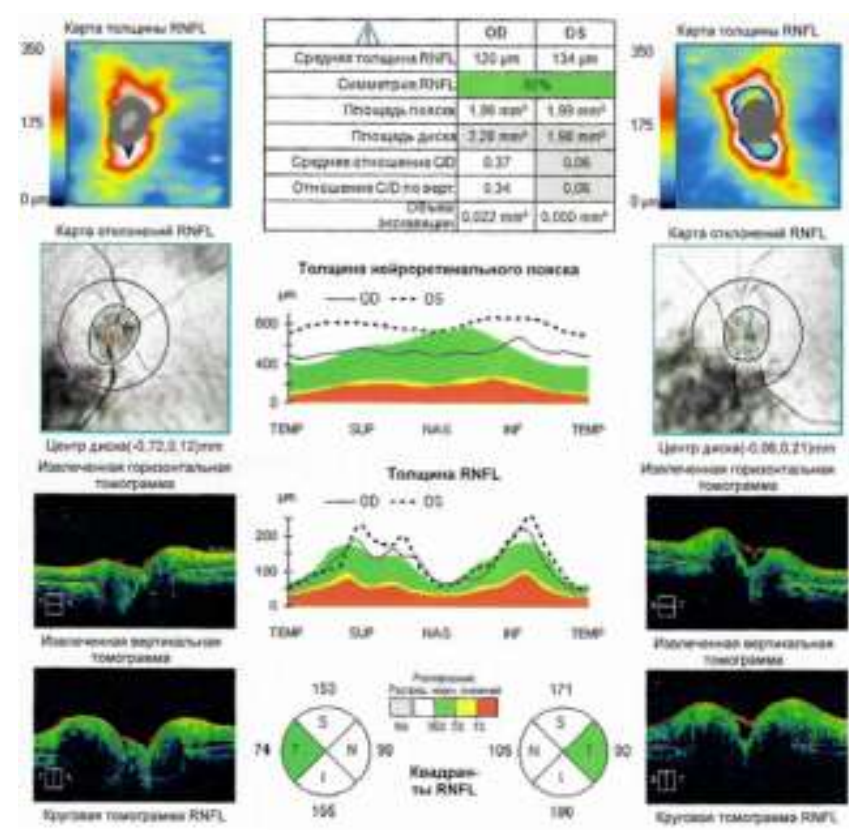

Figure 2. OCT of the disc of the optic nerve OU: the thickness of the layer of nerve fibres is in the upper and lower nasal segments. $\mathrm{OS}$ - the prominence of the disc of the optic nerve in vitreous.

It was not possible to obtain the high-resolution images on the optical coherence tomography with the function of the angiography above $7 / 10$ due to the absence of a central fixation.

The fascinated changes of the cerebral cortex have not been found during the MRT. The diagnosis was made OU: Acute optic neuropathy of toxic genesis.

A course of integrated conservative treatment has been introduced: the anti-inflammatory, nootropic, metabolic, vitamin therapy, as well as magnetotherapy, electrostimulation and electrophoresis.

\section{Discussion}

Intoxication of the medical preparations is the most frequent of domestic poisoning, the main reasons for which may be: an improper storage of medicines, a selfmedication, a taking the several preparations at the same time, the mistaken medications, as well as the deliberate taking drugs caused by the conflict situations.

The signs of the poisoning of the medicines depend on the pharmacological group, the dose of taking the drug, the individual sensitivity, the elapsed time since taking the drug, the accompanying diseases. Closantel refers to the derivative of the salicylanilide, widely used as an anthelmintic preparation in veterinary practice. ${ }^{8}$ Several halogenated salicylanilides were synthesized with powerful anti-parasitic activity. ${ }^{9}$

Closantel is the active substance of the Closatrem, is active against the larval and the mature phases of nematode, including Haemonchus spp., Nematodirus spp., Trichostrongylus spp., Ostertagia spp., Oesophagostomum radiatum, Bunostomum spp., Chabertia ovis, the mature and the immature trematodes Fasciola hepatica, the mature trematodes Fasciola gigantica, and also the larvaes of the gadflies Hypoderma bovis, Hypoderma lineatum и Oestrus ovis, parasitizing in the cattle and in small ruminants. ${ }^{4}$
The most frequent symptoms after the closantel intoxication by animals include the loss of appetite, the ataxia or the dissenting movements, the weakness, the visual impairments and the blindness. ${ }^{11}$ The induced histopathological changes in the optic nerve after the overdose of the closantem include a significant sponge change, a swelling and the vacuum of the myelin leading to the atrophy of the disc of the optic nerve.

It leads to a swelling in the entracellular part of the optic nerve, which, while in the bone part, is shrinked, leading to the loss of the myelized axons in the retina, and the external layers, especially the photoreceptors are destructed and leading to the apoptosis later. ${ }^{10}$

The case of an erroneous prescription of closantel as the treatment for endometritis to the eleven patients by a gynecologist was described in 1993 in Lithuania. ${ }^{4}$ As a result of the ophthalmological survey after the drug intoxication by patients there were found: yet painless reduction in vision, a narrowing of the fields of view and a reduction in the thickness of the external and internal layers of the retina.

A rare case of the closantel poisoning of a 5-year-old girl with the manifestation of the complications in the form of the blindness, an increased liver enzymes and the coagulopathy was also described. ${ }^{3}$

\section{Conclusion}

The case of a bilateral acute optic neuropathy of toxic genesis in a patient by the unintentional application of a veterinary preparation with powerful anti-parasitic activity is presented in the report. We found that toxic damage of the ganglion cells of the retina was initially occurring, resulting in a reduction of the central visual acuity, followed by the development of the optic nerve atrophy.

Care must be exercised when dealing with medicines. Potentially, any medicines are toxic and, if used improperly, may cause a number of side effects from the visual and central nervous system.

The outcome of the disease and the effectiveness of treatment of toxicological patients depend largely on the timely started therapy, its volume and the strict continuity in the various stages of the application of detoxification and symptomatic therapy.

The authors declare that there is no conflict of interests regarding the publication of this paper.

\section{Acknowledgement}

IThe authors would like to thank the patient, S.N. Fedorov NMRC, MNTK, Eye Microsurgery, Russia for facilitating this research.

\section{References}

1. Badrane N, Abbada A, Chaoui H, Aoued L, Rhalem N, Benjelloun BS, Bencheikh RS. Blindness following closantel poisoning: Report of three cases. XXXIII International Congress of the European Association of Poisons Centres and Clinical Toxicologists 
(EAPCCT) 28-31 May 2013, Copenhagen, Denmark. Clin Toxicol (Phila); 2013. 51:280.

2. Borges AS, Mendes LC, de Andrade AL, Machado GF, Peiro JR. Optic neuropathy in sheep associated with overdosage of closantel. Vet Hum Toxical; 1999 Dec; 41 (6):378-80. Avalaible form: https://pubmed.ncbi.nlm.nih.gov/10592945/

3. Essabar L, Meskini T., Ettair S, Erreimi N, Mouane N. Harmful use of veterinary drugs: Blindness following closantel poisoning in a 5-year-old girl. Asia Pac J Med Toxicol; 2014. 3:173-5.

DOI: 10.22038/APJMT.2014.3486

4. Hoen E, Hodgkin C. Harmful human use of donated veterinary drug. Lancet; 1993; 342(8866):308-9.

5. Ioyleva EE. Diagnostic value of the computer colorimetric analysis method in predicting the restoration of visual functions in toxic lesions of the optic nerve. Ophthalmosurgery; 2003. 1:40-43.

6. Koziolek MJ, Patschan D, Desel H, Wallbach M, Callizo J. Closantel Poisoning Treated With Plasma Exchange. JAMA Ophthalmology; 2015. 133(6):71820. DOI: 10.1001/jamaophthalmol.2015.191
7. Plumb D. Veterinary drug handbook. 3rd ed. Ames: Iowa State University Press; 1999. 411-412.

8. Safonenko A, Yu, Ioyleva EE. Cutting-edge visualization technologies in the diagnostics of optic nerve pathology. Practical Medicine; 2018. 3(114):156-159.

9. Swan GE. The pharmacology of halogenated salicylanilides and their anthelmintic use in animals. Journal of the South African Veterinary Association 2; 1999. 61 - 70. DOI: 10.4102/jsava.v70i2.756

10. Van der Lugt JJ, Venter I. Myelin vacuolation, optic neuropathy and retinal degeneration after closantel overdosage in sheep and in a goat. J Comp Pathol; 2007. 136(2-3):87-95.

DOI: $10.1016 /$ j.jcpa.2006.11.007

11. Venkatesh R., Pereira A, Aseem A, Yadav N.K. Commentary: Closantel - A lesser-known evil. Indian J Ophthalmol; 2019. 67:1771-2. Avalaible from: https://www.ijo.in/article.asp?issn=0301 4738; year $=2019 ;$ volume $=67 ;$ issue $=10 ;$ spage $=1771 ;$ ep age $=1772$; aulast $=$ Venkatesh 